\title{
Micro- and Macrostresses in Two Level Model of Coating Growth
}

\author{
N. N. Nazarenko ${ }^{1, \text { a) }}$ and A. G. Knyazeva, ${ }^{1,2, b)}$ \\ ${ }^{1}$ Institute of Strength Physics and Materials Science SB RAS, Tomsk, 634055, Russia \\ ${ }^{2}$ National Research Tomsk Polytechnic University, Tomsk, 634050, Russia \\ a) Corresponding author: nnelli@ispms.tsc.ru \\ b)anna@ispms.tsc.ru
}

\begin{abstract}
In the work, a two level model of coating growing with the diffusion and chemical compounds formation is proposed. The process of coating formation includes different physico-chemical steps and transformations of the structure. From the experiments it was established that the coating consists of the following substances: 4+ titanium oxide, titanium pyrophosphate, calcium pyrophosphate, calcium titanophosphate. Coating growth rate is determined by the deposition rate and the dispersion of the particles suspended in the electrolyte. The processes taking place in the electrolyte itself are not considered. The two levels (level of separate particles and level of macroscopic sample) are connected through the calculation of effective properties and parameters of the reaction. The influence of the model's parameters on the coating compound's and the sample's stress-strain state has been investigated.
\end{abstract}

Keywords: coating growing, spherulite, mechanical stresses

\section{INTRODUCTION}

Coating growth under microarc oxidation is a rather complex process. Experimentally it is not possible to describe this process dynamically but it can be done using a mathematical model implemented numerically. The content of calcium and phosphorus in the coating should be close in composition with the bone tissue. A coating deposition experiment can be performed in different ways. The technological parameters of coating depositions (voltage, time of coating formation, electrolyte composition) influence coating's properties: thickness, mass, porosity and coating composition. The coating's thickness and spherulite size growths with voltage increases. Taking into account the known facts, we suggest a model describing coating growth and chemical composition formation in the structural elements. As a result, on different levels, we have mechanical stresses that influence the final product's quality.

\section{FORMULATION OF THE PROBLEM}

The problem of coating formation via the microarc oxidation method includes different physical and chemical stages as well as structure transformations. Thus, from the experiment [1] it is deduced that a coating with spherulites as structural elements contains the following substances: 4+ titanium oxide, titanium pyrophosphate, calcium pyrophosphate, calcium titanophosphate. The chemical compounds are presumably formed in the bulk of the growing coating and this process is accompanied by the diffusion of the elements coming from the electrolyte. The deposition rate of the dispersed particles contained in the electrolyte suspension can be considered to be predetermined. Diffusion and chemical reactions occur at different structural levels: in the pores between the spherulites and in the bulk of the particles. Previous theoretical investigations $[2,3]$ of coating growth did not analyze the structural features. The distribution of the concentration of elements and phases as well as that of mechanical stresses and strains is apparent from the model. To take into account the formation of the growing coating's structure, the underlying mathematical model $[2,3]$ was supplemented by the description of the processes in the bulk of individual spherulites (Fig. 1).

International Conference on Physical Mesomechanics of Multilevel Systems 2014

AIP Conf. Proc. 1623, 431-434 (2014); doi: 10.1063/1.4898974

(C) 2014 AIP Publishing LLC 978-0-7354-1260-6/\$30.00 


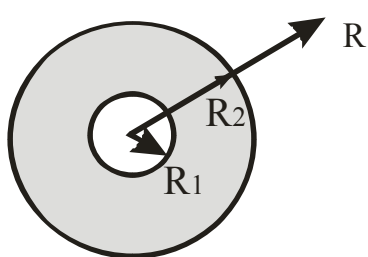

FIGURE 1. Illustration to problem definition for spherulites

It is assumed, that the formation of an individual layer of spherulites occurs discretely through some time interval determined by the experiment's conditions. The size of the spherulite layer and hence the spherulites' average inner and outer radius is associated with particular experiments we conducted. Before the formation of the first layer, for example, over time $t=\Delta t_{\mathrm{c}}$ we have a usual diffusion problem with moving the outer boundary at a given velocity as in $[2,3]$. It includes the usual equations for the diffusion in the substrate and in the coating, with different diffusion factors for the substances and ions coming from the electrolyte (water, calcium, phosphate, hydrogen and hydroxyapatite). In the center of the substrate, we have a symmetry condition; at the interface between the substrate and the coating, the conditions of diffusion fluxes' and chemical potentials' equality are assumed; the concentrations are provided for the outer boundary of the growing coating. Unlike previous studies [2, 3], all sources and links in the equations for the macro-level are absent.

After the time of $\Delta t_{\mathrm{c}}$ the spherulite layers form, and the diffusion and chemical reactions occur in them. This sub-problem is formulated in the local spherical coordinates system. On the outer boundary of the outer spherulite layer, concentration $\mathrm{C}_{\mathrm{ie}}$ is specified for the substances incoming from the electrolytic solution: water, calcium, phosphate, hydrogen, hydroxyapatite.

Four compounds form in the coating: $4+$ titanium oxide, calcium pyrophosphate, titanium pyrophosphate, calcium titanophosphate. These compounds can be obtained presumably by the following general reaction:

$$
\begin{gathered}
\mathrm{Ti}+2 \mathrm{H}_{2} \mathrm{O} \stackrel{k_{1}}{\rightarrow} \mathrm{TiO}_{2}+2 \mathrm{H}_{2}, \\
6 \mathrm{Ca}+6 \mathrm{PO}_{4}+3 \mathrm{H}_{2} \stackrel{k_{2}}{\rightarrow} 3 \mathrm{Ca}_{2} \mathrm{P}_{2} \mathrm{O}_{7}+3 \mathrm{H}_{2} \mathrm{O}, \\
\mathrm{Ti}+2 \mathrm{PO}_{4}+\mathrm{H}_{2} \stackrel{k_{3}}{\rightarrow} \mathrm{TiP}_{2} \mathrm{O}_{7}+\mathrm{H}_{2} \mathrm{O}, \\
\mathrm{Ca}_{10}\left(\mathrm{PO}_{4}\right)_{6}(\mathrm{OH})_{2}+4 \mathrm{Ti}+\mathrm{H}_{2} \stackrel{k_{4}}{\rightarrow} \mathrm{CaTi}_{4}\left(\mathrm{PO}_{4}\right)_{6}+9 \mathrm{Ca}+2 \mathrm{H}_{2} \mathrm{O} .
\end{gathered}
$$

As a result, the diffusion-kinetic problem for the spherulites includes the diffusion and chemical kinetics equations in the spherical coordinate system:

$$
\begin{gathered}
\frac{\partial y_{i}}{\partial t}=\frac{1}{r^{2}} \frac{\partial}{\partial r}\left(r^{2} D_{p i} \frac{\partial y_{i}}{\partial r}\right)+\sigma_{i}, \quad i=1-5, \\
\frac{\partial y_{i}}{\partial t}=\sigma_{i}, \quad i=6-9, \\
r=R_{1}: \frac{\partial y_{i}}{\partial r}=0, \quad i=1-5, \\
r=R_{2}: \quad y_{i}=C_{i e}, \quad i=1-5, \\
t=0: \quad y_{i}=0, \quad i=1-9
\end{gathered}
$$

TABLE 1. Concentrations designation

\begin{tabular}{cccc}
\hline $\begin{array}{c}\text { Designation of } \\
\text { Concentrations in the } \\
\text { Micro/Macro Problem }\end{array}$ & Compound/Ion & $\begin{array}{c}\text { Designation of } \\
\text { Concentrations in the } \\
\text { Micro/Macro Problem }\end{array}$ & Compound/Ion \\
\hline$y_{1} / C_{1}$ & $\mathrm{H}_{2} \mathrm{O}$ & $y_{6} / C_{6}$ & $\mathrm{TiO}_{2}$ \\
\hline$y_{2} / C_{2}$ & $\mathrm{Ca}^{2+}$ & $y_{7} / C_{7}$ & $\mathrm{Ca}_{2} \mathrm{P}_{2} \mathrm{O}_{7}$ \\
\hline$y_{3} / C_{3}$ & $\mathrm{PO}_{4}^{3-}$ & $y_{8} / C_{8}$ & $\mathrm{TiP}_{2} \mathrm{O}_{7}$ \\
\hline$y_{4} / C_{4}$ & $\mathrm{H}_{2}$ & $y_{9} / C_{9}$ & $\mathrm{CaTi}_{4}\left(\mathrm{PO}_{4}\right)_{6}$ \\
\hline$y_{5} / C_{5}$ & $\mathrm{Ca}_{10}\left(\mathrm{PO}_{4}\right)_{6}(\mathrm{OH})_{2}$ & $y_{10} / C_{10}$ & $\mathrm{Ti}$ \\
\hline
\end{tabular}


where $\sigma_{i}=\sum_{k=1}^{r} m_{i} v_{i k} \phi_{k}, \quad y_{i}$ are the mass concentrations of chemical compounds or ions in the spherulites (designations are given in Table 1), $D_{p i}$ is the effective diffusion factor in the spherulite of the $i$-th compound or ion, $\phi_{k}$ is the reaction rate obeying the law of mass action, $m_{i}$ are molar masses, $\rho_{\mathrm{c}}$ is the density of the coating.

The next layer of spherulites forms through time $t=2 \Delta t_{\mathrm{c}}$.

Now, at the boundary $r=R_{2}$ for the spherulite from the first layer, average concentrations of $\mathrm{i}$-th elements are given from the upper layer (2), and the boundary conditions for spherulites from the upper layer are formulated by the analogy to the previous one. Average composition of the coating is derived from average concentrations:

$$
C_{i}=\frac{1}{R_{2}-R_{1}} \int_{R_{1}}^{R_{2}} y_{i} \mathrm{~d} r, \quad i=1-9 .
$$

Furthermore, the growth of the coating and its average composition is modeled in a similar way.

In the substrate, the usual diffusion problem is solved.

The evaluation of mechanical stresses in the spherulites presents considerable practical interest due to the presence of concentration gradients in them. To find the distribution of stresses in the spherulites using a given distribution of concentrations, it is required to solve the problem of the mechanical equilibrium of an individual spherulite. A similar problem is solved in the theory of thermoelasticity [5] for the case of spherical symmetry. By replacing $\alpha_{T}\left(T-T_{0}\right)$ with $w=3 \sum_{i=1}^{9}\left(\alpha_{i}-\alpha\right)\left(y_{i}-y_{i 0}\right)$ in a known solution, we obtain

$$
\sigma_{r}=\frac{6 E}{1-v}\left[\frac{r^{3}-R_{1}^{3}}{r^{3}\left(R_{2}^{3}-R_{1}^{3}\right)} \int_{R_{1}}^{R_{2}} w r^{2} \mathrm{~d} r-\frac{1}{r^{3}} \int_{R_{1}}^{r} w r^{2} \mathrm{~d} r\right], \quad \sigma_{\Theta}=\frac{6 E}{1-v}\left[\frac{2 r^{3}-R_{1}^{3}}{2 r^{3}\left(R_{2}^{3}-R_{1}^{3}\right)} \int_{R_{1}}^{R_{2}} w r^{2} \mathrm{~d} r+\frac{1}{2 r^{3}} \int_{R_{1}}^{r} w r^{2} \mathrm{~d} r-\frac{w}{2}\right],
$$

where $E$ is the elasticity modulus, $v$ is Poisson ratio, $\alpha_{i}$ and $\alpha$ are the expansion concentration factors calculated similarly to $[3,6]$.

In general, obviously, the elastic modulus is some function of composition. However, such dependences are unknown from the experiment. There are no theoretical approaches to effective mechanical properties calculation for such materials. In this paper, it is assumed that (on average) the elastic modulus is $E=1 \cdot 10^{10} \mathrm{~Pa}$ for tricalcium phosphate and hydroxyapatite.

\section{NUMERICAL METHOD}

The problem is solved numerically by the method described in $[2,3]$. The physical properties are shown ibid.
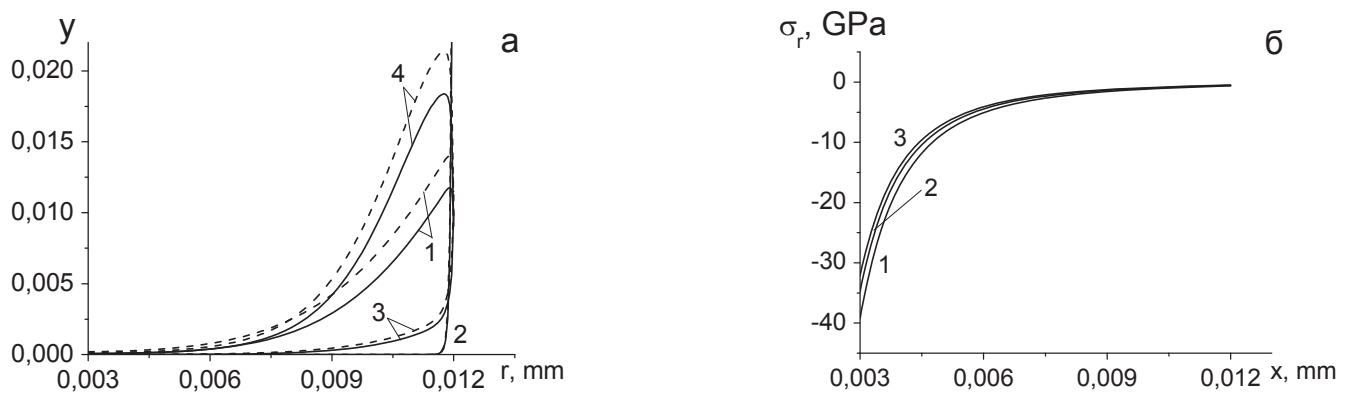

FIGURE 2. Distribution of concentrations $\mathrm{TiO}_{2}(1), \mathrm{Ca}_{2} \mathrm{P}_{2} \mathrm{O}_{7}$ (2), $\mathrm{TiP}_{2} \mathrm{O}_{7}$ (3) and $\mathrm{CaTi}_{4}\left(\mathrm{PO}_{4}\right)_{6}$ (4) in the first (solid line)

and the second (dotted line) layers of spherulite for time point $t=250 \mathrm{~s}$ (a) and the distribution of radial stresses in the spherulites in the middle layer for times: $250(1), 285(2), 530 \mathrm{~s} \mathrm{(3)} \mathrm{(b);} R_{1}=0.003, R_{2}=0.012$,

$$
D_{\mathrm{c}}=10^{-7}, D_{\mathrm{p}}=10^{-7}, K_{1}=3.98 \cdot 10^{-8}, \mathrm{~K}_{2}=5.3 \cdot 10^{13}, \mathrm{~K}_{3}=3 \cdot 10^{-5}, \mathrm{~K}_{4}=5 \cdot 10^{-6}
$$




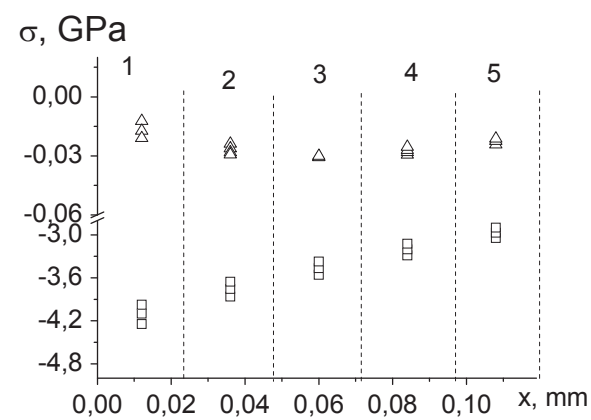

FIGURE 3. Average radial (squares) and tangent (triangles) stresses in five layers of coating

According to the experiment [1], when the voltage difference of the process is $250 \mathrm{~V}$, the coating thickness is $0.072 \mathrm{~mm}, R_{1}=0.003 \mathrm{~mm}, R_{2}=0.012 \mathrm{~mm}$. Therefore, three of the spherulite layers (the first is nearer to the substrate, the second is in the center of the coating and the third is closer to the electrolyte) are formed in such a coating's thickness. Some results are presented below.

The distribution of the concentration of the $\mathrm{TiO}_{2}, \mathrm{Ca}_{2} \mathrm{P}_{2} \mathrm{O}_{7}, \mathrm{TiP}_{2} \mathrm{O}_{7}$ and $\mathrm{CaTi}_{4}\left(\mathrm{PO}_{4}\right)_{6}$ chemical compounds is different in spherulites from different layers as seen from Fig. 2(a). Moreover, the distribution of concentration changes over time. Radial tension decreases in modulus over time near the inner surface of a spherulite and near the outer surface it varies slightly. For the radial component of the stress, the tensor in the spherulite of the middle layer can be seen in Fig. 2(b). The absolute value of radial tension decreases in time near the inner surface of a spherulite and varies slightly near the outer surface.

The average concentrations correspond to the average stresses in the layers of spherulites, which are also found by averaging (Fig. 3). The vertical dashed lines mark the area where the spherulites are located.

It follows from Figure 3 that the average radial stresses decrease in modulus and the average shear stresses of up to three layers of spherulites increase in absolute value, and then decrease. The electrolytic composition affects the content of the substances in the coating and consequently changes the stress components that are associated with theses changes. However, the stresses in the coating layers are practically unchanged for various electrolyte compositions.

\section{SUMMARY}

Thus, in the work a two level model of coating growth with the diffusion and spherulitic structure formation is proposed. The distributions of concentration of the deposited components and the chemical compounds that form in the spherulites as well as their average content in the coating layers have been calculated. The mathematical model can provide a forecast for coating deposition times other than those known from the experiment. The distribution of radial and tangential stresses in the spherulites, and the average stresses in the coating have been calculated.

This work has been performed within the frame of the program for fundamental research of SB RAS, project III.23.2.5.

\section{REFERENCES}

1. Yu. P. Sharkeev, E. V. Legostaeva, I. A. Khlusov, K. S. Kulyashova, and E. G. Komarova, Phys. Chem. Mater. Treatment 2, 51 (2012).

2. N. N. Nazarenko and A. G. Knyazeva, Phys. Chem. Mater. Treatment 4, 97 (2011).

3. N. N. Nazarenko and A. G. Knyazeva, Fiz. Mezomekh. 14(5), 71 (2011).

4. N. N. Nazarenko and A. G. Knyazeva, Math. Model. 21(1), 92 (2009).

5. S. P. Timoshenko and J. N. Goodier, Theory of Elasticity (Nauka, Moscow, 1975).

6. N. N. Nazarenko and A. G. Knyazeva, Fiz. Mezomekh. 13(3), 95 (2010). 